\title{
Ansiedade e depressão no contexto da pandemia COVID-19 e a relação com os mecanismos de defesa das gestantes
}

\author{
Anxiety and depression in the COVID-19 pandemic context and the relationship with the defense \\ mechanisms of pregnant women
}

Ansiedad y depresión en el contexto de la pandemia COVID-19 y la relación com los mecanismos de defensa de la gestante

Recebido: 20/07/2021 | Revisado: 29/07/2021 | Aceito: 31/07/2021 | Publicado: 06/08/2021

\author{
Isabela Pechinim \\ ORCID: https://orcid.org/0000-0002-3927-9323 \\ Faculdade de Medicina de São José do Rio Preto, Brasil \\ E-mail: isabelapechinim@gmail.com \\ Giovanna Albéfaro Sandonato Barbosa \\ ORCID: https://orcid.org/0000-0003-4730-7360 \\ Faculdade de Medicina de São José do Rio Preto, Brasil \\ E-mail: albefarosandonato@gmail.com \\ Alexandre Lins Werneck \\ ORCID: https://orcid.org/0000-0002-2911-8091 \\ Faculdade de Medicina de São José do Rio Preto, Brasil \\ E-mail: alexandre.werneck@famerp.br
}

\begin{abstract}
Resumo
Introdução: O período gravídico é evidenciado por modificações, que incluem alterações físicas, psicológicas e sociais em gestantes. Objetivo: Verificar a ansiedade e a depressão no contexto da pandemia e a relação com os mecanismos de defesa das gestantes para que estratégias de enfrentamento com assistência profissional sejam elaboradas. Método: Estudo transversal com delineamento descritivo, abordagem quantitativa do tipo analítica, com correlação entre variáveis, desenvolvido em um Centro Especializado Materno Infantil (CEMI) e no setor de emergência obstétrica de um hospital, ambos no interior de São Paulo. Participaram do estudo 61 gestantes, sendo 30 do CEMI e 31 da emergência obstétrica, com idade igual ou maior a 18 anos. Os dados foram coletados por meio de dois questionários, sendo um com dados sociodemográficos e outro com questões abertas e fechadas sobre mecanismos de defesa positivos e negativos. Para avaliar os sintomas de ansiedade e depressão, foi utilizado a Escala Hospitalar de Ansiedade e Depressão, dados que foram analisados de forma descritiva e inferencial. Resultados: Constatou-se que $29,51 \%$ das gestantes apresentaram sintomas de ansiedade e $24,59 \%$ sintomas de depressão. Das gestantes que apresentaram depressão, 66,67\% encontravam-se na emergência obstétrica. Verificou-se que 14,75\% afirmaram que consumiram bebida alcoólica ou drogas ilícitas no período de pandemia COVID-19, 45,90\% das gestantes não realizaram atividade física no período da pandemia e 49,18\% tiveram dificuldade para dormir. Conclusão: Os resultados do estudo mostram que parte das gestantes apresentam uma situação de vulnerabilidade relacionada à saúde mental prejudicada e alterações na rotina acentuadas no atual período pandêmico.
\end{abstract}

Palavras-chave: Ansiedade; Depressão; Gestantes; COVID-19.

\begin{abstract}
Introduction: The pregnancy period is evidenced by changes, which include physical, psychological and social changes in pregnant women. Objective: To verify anxiety and depression in the COVID-19 pandemic context and the relationship with the defense mechanisms of pregnant women so that coping strategies with professional assistance are developed. Method: Cross-sectional study with descriptive design, quantitative analytical approach, with correlation between variables, developed in a specialized maternal and child center and in the emergency obstetric sector of a hospital, both in the São Paulo countryside. Sixty-one pregnant women participated in the study, 30 from specialized maternal and child center and 31 from the obstetric emergency, aged 18 years or over. Data were collected through two questionnaires, one with sociodemographic data and the other with open and closed questions about positive and negative defense mechanisms. To assess symptoms of anxiety and depression, the Hospital Anxiety and Depression Scale was used, data that were analyzed descriptively and inferentially. Results: It was found that $29.51 \%$ of pregnant women had symptoms of anxiety and $24.59 \%$ symptoms of depression. Of the pregnant women who presented depression, $66.67 \%$ were in the obstetric emergency. It was found that $14.75 \%$ said they consumed alcohol or illicit drugs during the COVID-19 pandemic context, $45.90 \%$ of the pregnant women did not perform physical
\end{abstract}


activity during the pandemic period and $49.18 \%$ had difficulty sleeping. Conclusion: The results of the study show that part of the pregnant women have a situation of vulnerability related to impaired mental health and marked changes in routine in the current pandemic period.

Keywords: Anxiety; Depression; Pregnant women; COVID-19.

\section{Resumen}

Introducción: El período de embarazo se evidencia por cambios, que incluyen cambios físicos, psicológicos y sociales en la gestante. Objetivo: Verificar la ansiedad y depresión en el contexto de la pandemia y la relación con los mecanismos de defensa de la gestante para que se desarrollen estrategias de afrontamiento con asistencia profesional. Método: Estudio transversal con diseño descriptivo, abordaje analítico cuantitativo, con correlación entre variables, desarrollado en un centro especializado materno infantil (CEMI) y en el sector obstétrico de emergencia de un hospital, ambos del interior de São Paulo. En el estudio participaron 61 mujeres embarazadas, 30 del CEMI y 31 de la emergencia obstétrica, de 18 años o más. Los datos fueron recolectados a través de dos cuestionarios, uno con datos sociodemográficos y otro con preguntas abiertas y cerradas sobre mecanismos de defensa positivos y negativos. Para evaluar los síntomas de ansiedad y depresión se utilizó la Escala Hospitalaria de Ansiedad y Depresión, datos que fueron analizados de forma descriptiva e inferencial. Resultados: Se encontró que el 29,51\% de las gestantes presentaban síntomas de ansiedad y el 24,59\% síntomas de depresión. De las gestantes que presentaron depresión, el $66,67 \%$ se encontraba en la urgencia obstétrica. Se encontró que el 14,75\% dijo haber consumido alcohol o drogas ilícitas durante el período pandémico de COVID-19, el 45,90\% de las embarazadas no realizó actividad física durante el período pandémico y el 49,18\% tuvo dificultad para dormir. Conclusión: Los resultados del estudio muestran que parte de las mujeres embarazadas tiene una situación de vulnerabilidad relacionada con el deterioro de la salud mental y cambios marcados en la rutina en el período pandémico actual.

Palabras clave: Ansiedad; Depresión; Mujeres embarazadas; COVID-19.

\section{Introdução}

O período gravídico é evidenciado por modificações, que incluem alterações físicas, psicológicas e sociais em gestantes (Yan, Ding, \& Guo, 2020). A interface para analisar a saúde materna em âmbito biopsicossocial, mostra uma relação com o contexto da gravidez, a pandemia COVID-19 e o impacto negativo emocional das gestantes relacionado ao isolamento social e a dimensão dos desconfortos que podem apresentar, uma vez que o conceito de saúde de um indivíduo integra fatores ampliados (Celeumans, Hompes, \& Foulon, 2020). Entretanto, apesar das consequências para a saúde materna e fetal, o reconhecimento e tratamento no estudo e interpretação dos sinais e sintomas relacionados a saúde mental continua a apresentar lacunas (Dennis, Falah-Hassani, \& Shiri, 2017).

Ressalta-se que, transtornos mentais entre mulheres durante a gestação estão relacionados a fatores de riscos para o binômiomãe e feto (Arrais, Araujo, \& Schiavo, 2019). A ansiedade é evidenciada como um fator que pode desencadear Depressão Pós-Parto (DPP) (Arrais, Araujo, \& Schiavo, 2019), tal afirmação aborda a importância do acompanhamento e tratamento adequado no controle do transtorno. Caso ocorra negligência na assistência a gestantes e falha na orientação e apoio, aponta-se que 70\% das gestantes que apresentam DPP podem permanecem em sofrimento psíquico no período após o nascimento do filho e primeiros anos de vida (Lucci, Otta, David, \& Chelini, 2016).

Além disso, a depressão e ansiedade proporcionam um impacto negativo relacionada ao aumento do risco de aborto espontâneo, parto prematuro, baixo peso do recém-nascido e Apgar mais baixo (Grigoriadis et al., 2018). Dessa forma, a longo prazo, o estresse durante a gestação pode influenciar no déficit do desenvolvimento cognitivo e comportamental da criança, e como consequência, um risco elevado de afetar a saúde mental (Bergh, Dahnke, \& Mennes, 2018).

A prevalência de ansiedade em âmbito internacional, aponta para um percentual de 15,2\% em mulheres durante o período pré-natal e pós-natal (Dennis, Falah-Hassani, \& Shiri, 2017). Juntamente a isto, a depressão gestacional possui relação com o nível de desenvolvimento do país, ou seja, países desenvolvidos, como Estados Unidos da América, Inglaterra, Canadá e Suécia, possuem taxas de prevalência mais baixas, em torno de 10\% e 15\% (Biratu \& Demewoz, 2015). Em contrapartida, países em desenvolvimento, com um contexto social, político e econômico precário, como Brasil, Bangladesh, Paquistão e África do Sul, a estimativa é de 25\% (Biratu \& Demewoz, 2015). 
No contexto pandêmico, as gestantes correm maior risco de depressão, ansiedade, transtorno de estresse póstraumático e suicídio, decorrentes de fatores estressores, sofrimento psicológico, alteração na rotina e aumento de cuidados relacionado a sua proteção contra o vírus (Thapa, Mainali, Schwank \& Acharya, 2020). Portanto, é imprescindível verificar o impacto da pandemia COVID-19 nesse grupo vulnerável e quais os mecanismos de defesa e estratégias de enfretamento utilizadas, sendo negativas ou positivas, para que seja possível uma assistência humanizada e de qualidade, com foco na continuidade do cuidado e abordagem que proporcione o cuidado psicológico as gestantes. Assim, o objetivo deste estudo foi verificar a ansiedade e a depressão no contexto da pandemia e a relação com os mecanismos de defesa das gestantes para que estratégias de enfrentamento com assistência profissional sejam elaboradas reduzindo os prováveis efeitos prejudiciais.

\section{Metodologia}

Estudo transversal com delineamento descritivo, abordagem quantitativa do tipo analítica, com correlação entre variáveis. No método quantitativo, a coleta de dados quantitativos ou numéricos é por meio do uso de medições de grandezas e é possível obter por meio da metrologia, números com suas respectivas unidades. Com isso, geram conjuntos de dados que podem ser analisados por meio de técnicas matemáticas (Pereira, et al., 2018). O estudo foi desenvolvido no centro especializado materno infantil (CEMI) no interior de São Paulo, no período de novembro de 2020 a fevereiro de 2021. A população foi composta pelas gestantes em acompanhamento na respectiva clínica. Foi feita uma amostragem não probabilística em sequência, ou seja, participaram todas as gestantes com idade igual ou maior do que 18 anos. A pesquisa foi feita na plataforma Google, por meio do aplicativo Google Forms, contendo o Termo de Consentimento Livre e Esclarecido (TCLE). Os contatos com as gestantes foram fornecidos pela clínica e a pesquisadora enviou via aplicativo WhatsApp para todas as gestantes. Momento em que receberam uma mensagem, informando sobre a pesquisa e caso aceitassem participar, bastava acessar o link enviado e clicar na opção aceitar ou não. Caso o convite fosse aceito, teriam acesso ao questionário. Do contrário, receberiam uma mensagem de agradecimento e o formulário não era enviado. Foram excluídas da pesquisa 47gestantes que não responderam ao questionário.

Em virtude da falta de adesão das gestantes da clínica, solicitamos ao Comitê de Ética e Pesquisa (CEP), a inclusão de um hospital referência em atendimento a gestantes, localizado no noroeste paulista. A pesquisa foi realizada no setor de emergência. A população foi composta por gestantes com idade maior ou igual a 18 anos, em atendimento na unidade. A amostra foi não probabilística em sequência, ou seja, foram incluídas todas as gestantes de uma população acessível no período de dezembro de 2020 a fevereiro de 2021 e excluídas duas gestantes com idade menor a 18 anos e três que não responderam ao questionário. A pesquisa foi realizada na forma presencial, com a pesquisadora obedecendo a todos os protocolos de segurança, salvaguardando a si e as entrevistadas. A pesquisadora informava a gestante sobre a pesquisa, respondia as dúvidas e deixava um questionário em cada leito da unidade. A pesquisadora aguardava as gestantes responderem e recolhia o questionário. Portanto, a amostra total da pesquisa, realizada nas duas instituições, foi constituída por 61 gestantes, sendo 30 do centro especializado materno infantil e 31 da emergência obstétrica.

Foi utilizado um questionário autoaplicável elaborado pela pesquisadora com questões abertas e fechadas para a caracterização das participantes e análise de mecanismos de defesa positivos e negativos, contemplando as variáveis dos estudos. Para a avaliação de estratégias de enfrentamento relacionada a saúde mental foi empregada a Escala Hospitalar de Ansiedade e Depressão HADS.A Escala Hospitalar de Ansiedade e Depressão HADS é utilizada para mensurar sintomas sugestivos de ansiedade e depressão. É composta por 14 questões divididas em duas subescalas: HADS-Ansiedade (HADS-A), com sete questões para o diagnóstico do Transtorno de Ansiedade Leve e HADS-Depressão (HADS-D), com outras sete para o Transtorno Depressivo Leve. A escala de resposta varia entre zero e três pontos com escore máximo de 21 pontos por 
subescala. Os pontos de corte obtidos na literatura são de $\geq 8$ pontos para cada transtorno, propostos a partir de critérios teóricos e empíricos derivados de amostras clínicas.

O estudo foi aprovado pelo Comitê de Ética em Pesquisa da Faculdade de Medicina São José do Rio Preto (FAMERP), parecer $\mathrm{n}^{\circ} 4.264 .104$ de 08 de setembro de 2020. Todos as gestantes participantes assinaram o Termo de Consentimento Livre e Esclarecido após esclarecimentos sobre os procedimentos, objetivo e destino dos dados da pesquisa, com a garantia do sigilo dos dados, conforme a resolução 466/2012 do Conselho Nacional de Saúde do Ministério da Saúde, que dispõe as diretrizes e normas regulamentares da pesquisa envolvendo seres humanos. Foram utilizados métodos de Estatísticas Descritivas e métodos de Estatísticas Inferenciais, analisando-se questões de probabilidade de uma população com base nos dados da amostra.

Em alguns momentos, dada a necessidade, para melhor entendimento, foram usados os seguintes métodos: Média; Mediana; Moda; Desvio Padrão; Erro Padrão; Valor Máximo; Valor Mínimo e Significância. O teste de Komolgorov-Smirnov analisou a normalidade. Foram feitos testes de hipóteses, usando método de Correlação de Spearman e U de Mann-Whitney, em que se analisou o comportamento das correlações entre as variáveis analisadas e o grau de explicação da variável dependente em relação as variáveis independentes da amostra.

De maneira descritiva, foi traçado o perfil da amostra estudada, contemplando as variáveis analisadas e seus desdobramentos. Os dados foram replicados de forma absoluta e relativas nesta primeira parte. No âmbito inferencial, foi traçado como objetivo estatístico, a análise de independência e predição entre as variáveis propostas no escopo do trabalho. Para isso, utilizou-se, dentro dos padrões esperados, o teste Correlação de Spearman e U de Mann-Whitney.

Vale ressaltar, que os resultados de independência entre as variáveis propostas, se deram pela análise entre os valores de $\mathrm{P}$ (significância). Essa métrica é considerada científica. Todas as análises foram obtidas pelo Software SPSS Statistics (Versão 23) atreladas as funcionalidades da ferramenta Excel (versão 2.016).

\section{Resultados}

A amostra da pesquisa foi constituída na maioria por mulheres $>30$ anos, casadas, no terceiro trimestre de gestação e primigestas. (Tabela 1.)

Tabela 1. Caracterização das gestantes da pesquisa. São José do Rio Preto, SP, Brasil. 2021.

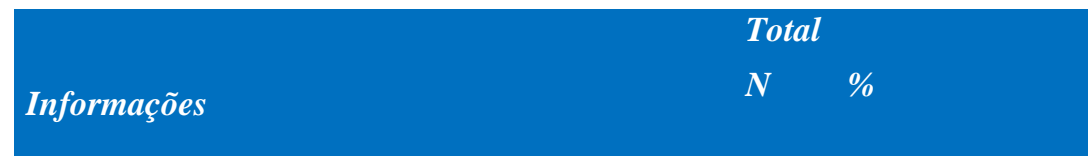

\section{Unidades}

$\begin{array}{lll}\text { Centro Materno Especializado Materno Infantil } & 30 & 49,18 \\ \text { Emergência Obstétrica } & 31 & 50,82 \\ \text { Total } & \mathbf{6 1} & \mathbf{1 0 0 , 0 0}\end{array}$

\section{Idade}

$\begin{array}{lll}\text { Até } 20 \text { anos } & 6 & 9,84 \\ 21 \text { a } 25 \text { anos } & 8 & 13,11 \\ 26 \text { a } 30 \text { anos } & 16 & 26,23 \\ >30 \text { anos } & 31 & 50,82 \\ \text { Total } & \mathbf{6 1} & \mathbf{1 0 0 , 0 0}\end{array}$




\section{Estado Civil}

$\begin{array}{lll}\text { Solteiro } & 13 & 21,31 \\ \text { Casado } & 41 & 67,21 \\ \text { União Estável } & 6 & 9,84 \\ \text { Divorciada } & 1 & 1,64 \\ \text { Total } & \mathbf{6 1} & \mathbf{1 0 0 , 0 0}\end{array}$

\section{Trimestre Atual}

$\begin{array}{lll}\text { Primeiro trimestre } & 5 & 8,20 \\ \text { Segundo trimestre } & 14 & 22,95 \\ \text { Terceiro trimestre } & 42 & 68,85 \\ \text { Total } & \mathbf{6 1} & \mathbf{1 0 0 , 0 0}\end{array}$

\section{Semana Gestacional}

$\begin{array}{lll}\text { Até } 15 \text { semanas } & 7 & 11,48 \\ 16 \text { a } 20 \text { semanas } & 7 & 11,48 \\ 21 \text { a } 25 \text { semanas } & 5 & 8,20 \\ 26 \text { a } 30 \text { semanas } & 3 & 4,92 \\ >30 \text { semanas } & 38 & 62,30 \\ \text { Missing } & 1 & 1,64 \\ \text { Total } & 61 & \mathbf{1 0 0 , 0 0}\end{array}$

\section{Gestação}

$\begin{array}{lll}\text { Primigesta } & 36 & 59,02 \\ \text { Secundigesta } & 11 & 18,03 \\ \text { Tercigesta } & 8 & 13,11 \\ \text { Multigesta } & 6 & 9,84 \\ \text { Total } & \mathbf{6 1} & \mathbf{1 0 0 , 0 0}\end{array}$

Fonte: Autores (2021).

A Tabela 2 demonstra mecanismos de defesa positivos e negativos que possuem impacto na saúde da gestante. A maioria relatou que recebeu visitas de familiares e amigos durante a pandemia e que não realizaram atividade física durante a pandemia. Houve um percentual significativo de gestantes que consumiram bebida alcoólica ou drogas ilícitas. 
Tabela 2. Mecanismos de defesa positivos e negativos utilizados por gestantes no contexto de pandemia COVID-19. São José do Rio Preto, SP, Brasil. 2021

\begin{tabular}{lll} 
& \multicolumn{2}{l}{ Total } \\
& $N$ & $\%$ \\
Informações & & \\
Recebeu visitas de familiares e amigos durante & \\
pandemia COVID-19 & & \\
Sim & 40 & 65,57 \\
Não & 21 & 34,43 \\
Total & $\mathbf{6 1}$ & $\mathbf{1 0 0 , 0 0}$
\end{tabular}

Consumiu bebida alcoólica ou drogas ilícitas

no período de pandemia COVID-19

$\begin{array}{lll}\text { Sim } & 9 & 14,75 \\ \text { Não } & 52 & 85,25 \\ \text { Total } & \mathbf{6 1} & \mathbf{1 0 0 , 0 0}\end{array}$

Realizou atividade física período de pandemia por COVID-19

\begin{tabular}{|c|c|c|}
\hline Sim, 1 vez por semana & 9 & 14,75 \\
\hline Sim, de 2 a 3 vezes por semana & 14 & 22,95 \\
\hline Sim, de 3 a 4 vezes por semana & 6 & 9,84 \\
\hline Sim, 5 ou mais & 4 & 6,56 \\
\hline Não realizei atividade física & 28 & 45,90 \\
\hline Total & 61 & 100,00 \\
\hline \multicolumn{3}{|l|}{$\begin{array}{l}\text { Recebeu informações sobre medidas de } \\
\text { prevenção e proteção contra o vírus? }\end{array}$} \\
\hline $\operatorname{Sim}$ & 56 & 91,80 \\
\hline Não & 5 & 8,20 \\
\hline Total & 61 & 100,00 \\
\hline \multicolumn{3}{|l|}{$\begin{array}{l}\text { Você teve dificuldade para dormir no período } \\
\text { de pandemia COVID-19? }\end{array}$} \\
\hline $\operatorname{Sim}$ & 30 & 49,18 \\
\hline Não & 31 & 50,82 \\
\hline Total & 61 & 100,00 \\
\hline $\begin{array}{l}\text { Você teve alterações na rotina alimentar } \\
\text { (perda do apetite ou comer mais do que esta } \\
\text { acostumada)? }\end{array}$ & & \\
\hline
\end{tabular}




$\begin{array}{lll}\text { Sim } & 24 & 39,34 \\ \text { Não } & 37 & 60,66 \\ \text { Total } & \mathbf{6 1} & \mathbf{1 0 0 , 0 0}\end{array}$

Fonte: Autores (2021).

Relacionado a classificação de ansiedade e depressão, verifica-se que a maioria das gestantes encontram-se sem sintomas. O percentual de sintomas é maior do local de aplicação emergência obstétrica. (Tabela 3)

Tabela 3. Distribuição das gestantes segundo os resultados da subescalas HADS- A e HADS-D e Local de aplicação. São José do Rio Preto, SP, Brasil. 2021.

\begin{tabular}{|c|c|c|c|c|c|c|c|}
\hline \multirow{4}{*}{ Informações } & \multicolumn{7}{|c|}{ Unidades } \\
\hline & \multicolumn{2}{|c|}{ Total } & \multicolumn{2}{|c|}{ CEMI } & \multicolumn{2}{|c|}{$\begin{array}{l}\text { Emergência } \\
\text { Obstétrica }\end{array}$} & \multirow[t]{3}{*}{$\begin{array}{l}\text { Valor } \\
P\end{array}$} \\
\hline & $N$ & $\%$ & $N$ & $\%$ & $\boldsymbol{N}$ & $\%$ & \\
\hline & 61 & 100,00 & 30 & 49,18 & 31 & 50,82 & \\
\hline \multicolumn{8}{|c|}{ Classificação Ansiedade } \\
\hline Sem Sintomas $(<8)$ & 43 & 70,49 & 24 & 80,00 & 19 & 61,29 & \multirow{2}{*}{0,113} \\
\hline Com Sintomas $(>=8)$ & 18 & 29,51 & 6 & 20,00 & 12 & 38,71 & \\
\hline \multicolumn{8}{|c|}{ Classificação Depressão } \\
\hline Sem Sintomas $(<8)$ & 46 & 75,41 & 25 & 83,33 & 21 & 67,74 & \multirow{2}{*}{0,163} \\
\hline Com Sintomas $(>=8)$ & 15 & 24,59 & 5 & 16,67 & 10 & 32,26 & \\
\hline
\end{tabular}

Sobre o percentual de gestantes com sintomas de ansiedade, é possível identificar que é maior na unidade de emergência obstétrica e em primigestas. (Tabela 4)

Tabela 4. Ansiedade relacionada a caracterização e mecanismos de defesa positivos e negativos.

\begin{tabular}{|c|c|c|c|c|c|c|c|}
\hline \multirow{3}{*}{ Informações } & \multicolumn{2}{|c|}{ Total } & \multicolumn{2}{|c|}{$\begin{array}{l}\text { Sem } \\
<8\end{array}$} & \multicolumn{2}{|c|}{$\begin{array}{l}\text { Com Sintomas } \\
>=8\end{array}$} & \multirow[t]{3}{*}{ Valor $P$} \\
\hline & $N$ & $\%$ & $N$ & $\%$ & $N$ & $\%$ & \\
\hline & 61 & 100,00 & 43 & 70,49 & 18 & 29,51 & \\
\hline \multicolumn{8}{|l|}{ Unidades } \\
\hline CEMI & 30 & 49,18 & 24 & 55,81 & 6 & 33,33 & \multirow{2}{*}{0,113} \\
\hline Emergência Obstétrica & 31 & 50,82 & 19 & 44,19 & 12 & 66,67 & \\
\hline \multicolumn{8}{|l|}{ Estado Civil } \\
\hline Solteiro & 13 & 21,31 & 6 & 13,95 & 7 & 38,89 & \multirow{4}{*}{0,218} \\
\hline Casado & 41 & 67,21 & 33 & 76,74 & 8 & 44,44 & \\
\hline União Estável & 6 & 9,84 & 3 & 6,98 & 3 & 16,67 & \\
\hline Divorciada & 1 & 1,64 & 1 & 2,33 & 0 & 0,00 & \\
\hline
\end{tabular}




\begin{tabular}{|c|c|c|c|c|c|c|c|}
\hline \multicolumn{7}{|l|}{ Gestação } & \multirow{5}{*}{$\mathbf{0 , 0 2 5}$} \\
\hline Primigesta & 36 & 59,02 & 29 & 67,44 & 7 & 38,89 & \\
\hline Secundigesta & 11 & 18,03 & 6 & 13,95 & 5 & 27,78 & \\
\hline Tercigesta & 8 & 13,11 & 7 & 16,28 & 1 & 5,56 & \\
\hline Multigesta & 6 & 9,84 & 1 & 2,33 & 5 & 27,78 & \\
\hline \multicolumn{8}{|c|}{ Possui Alguma Doença? } \\
\hline Sim & 12 & 19,67 & 4 & 9,30 & 8 & 44,44 & \multirow{2}{*}{0,001} \\
\hline Não & 49 & 80,33 & 39 & 90,70 & 10 & 55,56 & \\
\hline
\end{tabular}

Recebeu visitas de familiares $e$

amigos durante pandemia

COVID-19?

\begin{tabular}{llllllll} 
Sim & 40 & 65,57 & 26 & 60,47 & 14 & 77,78 & \multirow{2}{*}{0,201} \\
Não & 21 & 34,43 & 17 & 39,53 & 4 & 22,22 & \\
\hline
\end{tabular}

\begin{tabular}{llllllll}
\hline $\begin{array}{l}\text { Consumiu bebida alcoólica ou } \\
\text { drogas ilícitas no período de } \\
\text { pandemia COVID-19? }\end{array}$ & & & & & & & \\
Sim & 9 & 14,75 & 4 & 9,30 & 5 & 27,78 & 0,065 \\
Não & 52 & 85,25 & 39 & 90,70 & 13 & 72,22 & \\
\hline
\end{tabular}

Realizou atividade física

período de pandemia por

COVID-19?

$\begin{array}{llllllll}\text { Sim, 1 vez por semana } & 9 & 14,75 & 8 & 18,60 & 1 & 5,56 & \\ \text { Sim, de 2 a 3 vezes por semana } & 14 & 22,95 & 10 & 23,26 & 4 & 22,22 & \\ \text { Sim, de 3 a 4 vezes por semana } & 6 & 9,84 & 5 & 11,63 & 1 & 5,56 & 0,066 \\ \text { Sim, 5 ou mais } & 16 & 26,23 & 4 & 9,30 & 12 & 66,67 & \\ \text { Não realizei atividade física } & 16 & 26,23 & 16 & 37,21 & 0 & 0,00 & \end{array}$

Testou positivo para COVID-

19 ?

$\begin{array}{llllllll}\text { Sim } & 3 & 4,92 & 3 & 6,98 & 0 & 0,00 & 0,258 \\ \text { Não } & 58 & 95,08 & 40 & 93,02 & 18 & 100,00 & \end{array}$

Fonte: Autores (2021).

Destaca-se que o percentual de gestantes que apresentaram sintomas de depressão é maior na unidade de emergência Obstétrica, em primigestas e que não realizaram atividade física durante a pandemia COVID-19. (Tabela 5) 
Tabela 5. Depressão relacionada a caracterização e mecanismos de defesa positivos e negativos.

\begin{tabular}{|c|c|c|c|c|c|c|c|}
\hline \multirow{4}{*}{ Informações } & \multicolumn{6}{|c|}{ Classificação de Depressão } & \multirow{4}{*}{$\begin{array}{l}\text { Valor } \\
P\end{array}$} \\
\hline & \multicolumn{2}{|c|}{ Total } & \multicolumn{2}{|c|}{ Sem Sintomas $<8$} & \multicolumn{2}{|c|}{ Com Sintomas $>=8$} & \\
\hline & $N$ & $\%$ & $N$ & $\%$ & $N$ & $\%$ & \\
\hline & 61 & 100,00 & 46 & 75,41 & 15 & 24,59 & \\
\hline \multicolumn{8}{|l|}{ Unidades } \\
\hline CEMI & 30 & 49,18 & 25 & 54,35 & 5 & 33,33 & \multirow{2}{*}{0,163} \\
\hline Emergência Obstétrica & 31 & 50,82 & 21 & 45,65 & 10 & 66,67 & \\
\hline \multicolumn{8}{|l|}{ Estado Civil } \\
\hline Solteiro & 13 & 21,31 & 7 & 15,22 & 6 & 40,00 & \multirow{4}{*}{0,059} \\
\hline Casado & 41 & 67,21 & 33 & 71,74 & 8 & 53,33 & \\
\hline União Estável & 6 & 9,84 & 5 & 10,87 & 1 & 6,67 & \\
\hline Divorciada & 1 & 1,64 & 1 & 2,17 & 0 & 0,00 & \\
\hline \multicolumn{8}{|l|}{ Gestação } \\
\hline Primigesta & 36 & 59,02 & 31 & 67,39 & 5 & 33,33 & \multirow{4}{*}{$\mathbf{0 , 0 0 7}$} \\
\hline Secundigesta & 11 & 18,03 & 8 & 17,39 & 3 & 20,00 & \\
\hline Tercigesta & 8 & 13,11 & 5 & 10,87 & 3 & 20,00 & \\
\hline Multigesta & 6 & 9,84 & 2 & 4,35 & 4 & 26,67 & \\
\hline \multicolumn{8}{|l|}{ Possui Alguma Doença? } \\
\hline Sim & 12 & 19,67 & 7 & 15,22 & 5 & 33,33 & \multirow{2}{*}{0,130} \\
\hline Não & 49 & 80,33 & 39 & 84,78 & 10 & 66,67 & \\
\hline
\end{tabular}

Recebeu visitas de familiares e

amigos durante pandemia

COVID-19?

\begin{tabular}{llllllll} 
Sim & 40 & 65,57 & 30 & 65,22 & 10 & 66,67 & 0,920 \\
Não & 21 & 34,43 & 16 & 34,78 & 5 & 33,33 & \\
\hline
\end{tabular}

Consumiu bebida alcoólica ou drogas ilícitas no período de pandemia COVID-19?

\begin{tabular}{llllllll} 
Sim & 9 & 17,65 & 7 & 15,22 & 2 & 40,00 & 0,861 \\
Não & 42 & 82,35 & 39 & 84,78 & 3 & 60,00 & \\
\hline
\end{tabular}

Realizou atividade física período de pandemia por COVID-19?

$\begin{array}{lllllll}\text { Sim, 1 vez por semana } & 9 & 14,75 & 8 & 17,39 & 1 & 6,67 \\ \text { Sim, de 2 a 3 vezes por semana } & 14 & 22,95 & 10 & 21,74 & 4 & 26,67 \\ \text { Sim, de 3 a 4 vezes por semana } & 6 & 9,84 & 5 & 10,87 & 1 & 6,67 \\ \text { Sim, 5 ou mais } & 4 & 6,56 & 4 & 8,70 & 0 & 0,00\end{array}$




\begin{tabular}{lccccccc} 
Não realizei atividade física & 28 & 45,90 & 19 & 41,30 & 9 & 60,00 \\
\hline Testou positivo para COVID-19? & & & & & & & \\
Sim & 3 & 4,92 & 3 & 6,52 & 0 & 0,00 & 0,318 \\
Não & 58 & 95,08 & 43 & 93,48 & 15 & 100,00 &
\end{tabular}

Fonte: Autores (2021).

Por meio da análise do cruzamento inferencial, aplicando-se o teste de Correlação de Spearman, conclui-se que há tendência do local de unidade emergência obstétrica, com sintomas para ansiedade com correlação (0,205), porém essa conclusão não pode ser afirmada cientificamente, devido significância $(0,113)$. Juntamente a isto, conclui-se que há tendência do local unidade emergência obstétrica, com sintomas para depressão com correlação $(0,181)$, porém essa conclusão não pode ser afirmada cientificamente, devido significância $(0,163)$.

Aplicando-se o teste de Correlação de Spearman, conclui-se que há tendência de gestantes com sintomas de ansiedade e que possuem alguma doença, com correlação (-0,403), essa conclusão pode ser afirmada cientificamente, devido significância $(0,001)$.

Conclui-se que há tendência de gestantes com sintomas de ansiedade e que não receberam visitas de familiares e amigos durante pandemia daCovid-19 com correlação $(-0,166)$, porém essa conclusão não pode ser afirmada cientificamente, devido significância $(0,201)$.

O teste de Correlação de Spearman, mostrou que há tendência de gestantes com sintomas de ansiedade, e que testaram positivo para a Covid-19 com correlação $(0,147)$, porém essa conclusão não pode ser afirmada cientificamente, devido significância $(0,258)$.

Além disso, o teste de Spearman, concluiu que há tendência de com sintomas de depressão e gestantes que não receberam visitas de familiares e amigos durante pandemia da Covid-19 com correlação (-0,013), porém essa conclusão não pode ser afirmada cientificamente, devido significância $(0,920)$ e que há tendência de com sintomas depressivos e gestante que consumiram bebida alcoólica ou drogas ilícitas no período de pandemia da Covid-19 com correlação (0,023), porém essa conclusão não pode ser afirmada cientificamente, devido significância $(0,861)$.

Relacionado ao acesso à informação, o teste de Spearman, concluiu que há tendência de do local unidade emergência obstétrica, e gestante que não receberam informações sobre medidas de prevenção e proteção contra o vírus com correlação $(0,168)$, porém essa conclusão não pode ser afirmada cientificamente, devido significância $(0,191)$.

\section{Discussão}

Nesta pesquisa, a amostra foi constituída por 61 gestantes, sendo que 68,85\% apresentavam a gestação no terceiro trimestre e apenas $8,20 \%$ no primeiro. Dado que corrobora dois estudos, um realizado na Polônia, em que as gestantes que encontravam-se no terceiro trimestre apresentavam 51,00\% da amostra e no primeiro trimestre 5,20\% (Stepowicz, Wencka, Bieńkiewicz, Horzelski \& Grzesiak, 2020) e outro realizado em Cingapura, com 44,30\% no terceiro trimestre e $18,00 \%$ no primeiro (Lee, Loy, Yang, Chan, \& Tan, 2020).

$\mathrm{Na}$ avaliação relacionada aos mecanismos de defesa positivos e negativos utilizados por gestantes durante a pandemia, os resultados desta pesquisa apontaram para uma possível relação entre autocuidado, depressão e ansiedade. Dessa forma, foi possível analisar que $27,78 \%$ das gestantes da amostra que afirmaram que consumiram bebida alcoólica ou drogas ilícitas no período de pandemia por coronavírus, apresentavam sintomas de ansiedade. Já as gestantes que apresentavam sintomas de depressão, o percentual de uso de álcool e drogas ilícitas durante a pandemia foi maior (40,00\%). Considerando que esses 
percentuais são significativos, uma vez que pode resultar em riscos a saúde da gestante e do feto, faz-se necessário uma abordagem que analise esses comportamentos. Um estudo realizado em 2020, evidenciou um percentual maior no consumo, uma vez que, na atual pesquisa foi constatado que 14,75\% das gestantes consumiram álcool ou drogas ilícitas e no estudo do outro estado, o percentual foi de 40,00\% da amostra (Gonçalves et al., 2020). Esses dados corroboram a importância do rastreio nas gestantes, mas há divergências nas porcentagens, que podem ser explicadas devido ao local de aplicação diferente, sendo que a atual pesquisa foi realizada no estado de São Paulo, e o outro estudo, realizado em um estado do nordeste do Brasil.

No que diz respeito à frequência da realização de atividade física durante a pandemia, constatou-se que 45,90\% das gestantes não realizaram. Evidencia-se que 60,00\% das gestantes que não realizaram atividade física apresentaram sintomas depressivos. Esse déficit na realização de atividade física foi percebido por outros estudos, como um realizado na Espanha, com uma porcentagem significativa das gestantes (87,70\%)que diminuíram a prática durante a pandemia (Biviá-Roig et al., 2020), e outro, realizado nos Estados Unidos, relatou que gestantes que demonstraram alterações nos exercícios durante a pandemia, apresentaram escores de depressão mais altos comparado com aquelas que não tiveram alterações. Com isso, supõese que a capacidade de manter uma rotina de atividades físicas possa auxiliar na saúde mental materna como um mecanismo importante para minimizar os impactos negativos e interferir de forma benéfica ao feto e a mãe (Almeida, Portugal \& Assis, 2020).

Destaca-se a informação como estratégia no enfrentamento para amenizar os prejuízos ao indivíduo. É fundamental o acesso a conteúdo que aborde o motivo do cenário atual, a importância do isolamento social e distanciamento como ferramentas para evitar o contágio da doença (Pancani, Marinucci, Aureli \& Riva, 2020). Entretanto, 8,20\% das gestantes, nesta pesquisa, afirmaram que não receberam informações sobre medidas de prevenção e proteção contra o vírus. Em contrapartida, um estudo realizado na Alemanha, obteve percentuais menores sobre a falta de informação, sendo que $3 \%$ dos participantes apontaram a necessidade de ser mais informados e 0,6\% afirmaram que estavam 'muito mal" informados sobre a pandemia e a Covid-19 (Führer et al., 2020). Essa divergência entre os dados da nossa pesquisa e os da Alemanha, pode ser explicada pela diferença entre a população, uma vez que esta pesquisa foi realizada com gestantes do interior do Estado de São Paulo e o outro estudo foi realizado em participantes adultos do sexo masculino e feminino de Schleswig-Holstein, SaxôniaAnhalt e Berlim.

$\mathrm{Na}$ atual pesquisa, com referência às interações sociais, 65,57\% das gestantes receberam visitas de familiares e amigos durante a pandemia. Entende-se que o apoio social é reconhecido como um importante mecanismo de proteção durante a gravidez. Um estudo realizado na Irlanda, apontou que gestantes utilizaram a conexão com outros indivíduos como uma estratégia para redução do estresse durante a pandemia (Matvienko-Sikar et al., 2020). Isto reforça a importância das interações sociais nesse cenário. Entretanto, segundo as recomendações de distanciamento social preconizada, ressalta-se que é fundamental que este contato seja on-line, para reduzir os danos à saúde em âmbito biopsicossocial.

Verificou-se, nesta pesquisa, que 49,18\% das gestantes tinham alteração no padrão de sono. Entre os brasileiros adultos que não tinham problemas de sono antes da pandemia, 43,50\% passaram a apresentar o problema e, entre aqueles que relataram história prévia de problema de sono, 48,00\% tiveram o problema agravado. Dessa maneira, pode-se ressaltar que o contexto atual, por ser um fator estressor, contribui de forma negativa para o padrão de sono (Barros et al., 2020) como um indicador de saúde e sua qualidade. A quantidade e/ou a qualidade do sono estão envolvidas com a manifestação de diversas alterações cognitivas, psicológicas, imunológicas e metabólicas, e pode contribuir para o risco de eventos adversos no parto, além de exercer influência na saúde física e mental materna durante e após o parto (Christian, Carroll, Teti, \& Hall, 2019), interferindo, portanto, continuamente no padrão de bem-estar de um indivíduo, e por consequência, em sua qualidade de vida, sendo imprescindível sua manutenção de forma eficaz. 
É de extrema relevância considerar que situações de estresse resultantes da pandemia, podem gerar excessos e descontroles relacionados ao consumo de alimentos (Yilmaz \& Gökmen, 2020). Em relação ao padrão alimentar, na nossa pesquisa, foi possível identificar que, 39,34\% das mulheres tiveram alteração. Esse padrão ineficaz da alimentação, é demonstrado em outro estudo realizado com gestantes, que relataram um déficit no consumo de alimentos de fonte proteica, vegetal, em laticínios e cereais (Rodríguez-Bernaç et al., 2013). Corroborando esses resultados, outra pesquisa apontou que, dois terços das participantes apresentaram uma dieta sem os nutrientes necessários, portanto, fora do recomendado (Biviá-Roig et al., 2020). Além disso, estudos presumiram que as respostas relacionadas à saúde mental e à disseminação da Covid-19, podem ter elevado o risco de desenvolver comportamentos alimentares desequilibrados (Wang et al., 2020). Essas disfuncionalidades, durante a gravidez, podem predispor diversas complicações, como diabetes gestacional e pré-eclâmpsia, que são fatores de importância no cuidado integral à saúde da mãe e do feto (Budak et al., 2019 \& Salman, Shah, Ali, Aziz, \& Vitale, 2018).

Com relação a Escala de Ansiedade e Depressão Hospitalar, nossos resultados mostraram um enfrentamento ineficaz relacionado à ansiedade, uma vez que 29,51\% das gestantes apresentaram sintomas. Resultados esses que merece atenção, visto que estudos demonstraram o sofrimento psicológico como prejudicial à saúde materna, fetal e, futuramente, ao desenvolvimento da criança (Fan et al., 2021). Um estudo realizado com 209 gestantes mostrou que 26,80\% das gestantes apresentaram sintomas de ansiedade (Silva, Nogueira, Clapis, \& Leite, 2017), o que corrobora dados da atual pesquisa. Diante dos dados apresentados, é importante analisar a ansiedade em âmbito de pandemia, uma vez que as mulheres grávidas podem estar expostas a um sofrimento psicológico adicional, sendo necessário uma assistência humanizada e de redução de danos durante o pré-natal.

Esta pesquisa demonstrou que $16,67 \%$ das gestantes, apresentaram sintomas depressivos. Relacionado à depressão durante a pandemia, outros estudos indicaram um percentual maior, constatando que a prevalência das mulheres com sintomas durante a gravidez, foi de 37,00\% (Lebel, MacKinnon, Bagshawe, Tomfohr-Madsen \& Giesbrecht, 2020), e outro estudo, apresentou valores próximos a 30,00\% (Dong et al., 2020). É possível explicar esta discrepância entre os resultados, em virtude de os locais de aplicação serem diferentes, uma vez que a atual pesquisa foi desenvolvida em um Hospital e em um Centro Especializado Materno Infantil no interior do estado de São Paulo e as outras pesquisas foram realizadas no Canadá e na China, respectivamente.

$\mathrm{Na}$ emergência obstétrica, 66,67\% das gestantes apresentaram sintomas de depressão, enquanto, no CEMI, o percentual foi menor, sendo de 33,33\%. Dessa forma, é possível fazer o questionamento sobre se o atendimento integral a essas usuárias é feito de forma a garantir o atendimento psicológico adequado em emergências obstétricas, uma vez que estudos apontaram esse setor como necessitando de reavaliações constantes e monitoramento mensal, tendo discussão entre a gestão e os trabalhadores sobre as estratégias de enfrentamento para classificar o risco e melhorar o atendimento a gestantes (Figueiroa et al., 2017).

Em tempos da pandemia da COVID-19, somam-se aos sentimentos oriundos da gravidez, parto e pós-parto, aqueles advindos do enfrentamento de uma enfermidade pouco conhecida (Parra-Saavedra et al., 2020). Tal vivência está diretamente relacionada a sentimentos de incerteza e medo, podendo afetar a relação da mãe com o bebê, tornando mais intensos os sentimentos inerentes a gravidez. Neste sentido, destaca-se que, os profissionais encontram cada vez mais desafios para assistência do cuidado, sendo muito difícil assegurar suporte social e emocional adequado (Estrela, Silva. Cruz, \& Gomes, 2020). A partir dos dados apresentados fica claro que a criação de uma rede de apoio para as mulheres passando pelo processo de gestação, parto e puerpério em tempos de pandemia é essencial e tem como objetivo a redução dessas ansiedades (Estrela, Silva. Cruz, \& Gomes, 2020). 
As limitações do estudo foram o cenário da pandemia pela COVID-19, período em que as gestantes estavam e estão sob incertezas e desconhecimento sobre o impacto do vírus SARS-CoV2 e a dificuldade de adesão das gestantes no preenchimento dos questionários, tendo como consequência uma amostra reduzida. Porém, compreende-se que é um ponto de partida para outros estudos e para discussões que abordem a importância de repensar sobre a assistência estabelecida a gestantes em âmbito biopsicossocial.

\section{Conclusão}

Os resultados do estudo mostram que parte das gestantes se encontra em uma situação de vulnerabilidade relacionada à saúde mental prejudicada e alterações na rotina acentuadas no atual período pandêmico. Foi possível observar que, aspectos básicos do atendimento biopsicossocial devem fazer parte do serviço de saúde, com uma grande demanda no local de emergência obstétrica, ou seja, é importante que os profissionais da equipe multiprofissional identifiquem fatores de risco relacionado a saúde mental em mulheres gestantes para que estratégias de saúde durante o atendimento sejam traçadas com o intuito de reduzir o impacto ao binômio mãe e feto.

Desse modo, dentro de um contexto humanizado, acredita-se que esse estudo possa contribuir para uma reflexão sobre a necessidade de um suporte as gestantes por meio do conhecimento de seus mecanismos de defesa e estratégias de enfrentamento com a finalidade de oferecer um cuidado ideal a pacientes em gestação durante uma pandemia.

Como citado anteriormente, o levantamento de dados por meio de questionário on-line foi considerado um dificultador devido à falta de adesão, com isso, sugestões para posteriores estudos são a aplicação novamente dos questionários em contexto após pandemia COVID-19, para que seja possível analisar de forma ampla as correlações entre ansiedade, depressão e mecanismos de defesa das gestantes em uma população maior.

\section{Referências}

Almeida, M. O., Portugal, T. M. \& Assis, T. J. (2020, abril/junho). Gestantes e COVID-19: isolamento como fator de impacto físico e psíquico. Revista Brasileira de Saúde Materno Infantil, 20(2), 599-602. http://dx.doi.org/10.1590/1806-93042020000200015

Arrais, A., Araujo, T. \& Schiavo, R. (2019, maio/agosto) Depressão e ansiedade gestacionais relacionadas à depressão pós-parto e o papel preventivo do prénatal psicológico. Revista Psicologia e Saúde, 11(2), 23-34. https://doi.org/10.20435/pssa.v0i0.706

Barros, M., Lima, M., Malta, D., Szwarcwald, C., Azevedo, R., Romero, D. et al. (2020). Relato de tristeza/depressão, nervosismo/ansiedade e problemas de sono na população adulta brasileira durante a pandemia de COVID-19. Epidemiologia e Serviços de Saúde, 29 (4), e2020427.

Bergh, B. V., Dahnke, R. \& Mennes, M. (2018, agosto). Prenatal stress and the developing brain: risks for neurodevelopmental disorders. Development and Psychopathology, 30(3), 743-762.

Biratu, A. \& Demewoz, H. (2015, outubro). Prevalence of antenatal depression and associated factors among pregnant women in Addis Ababa, Etiópia: a cross-sectional study. Reproductive Health, 12(99). https://doi.org/10.1186/s12978-015-0092-x

Biviá-Roig, G., La Rosa, VL, Gómez-Tébar, M., Serrano-Raya, L., Amer-Cuenca, J., Caruso, S., et al. (2020). Analysis of te impact of the confinement resulting from COVID-19 on the lifestyle and psychological wellbeing of Spanish pregnant women: na internet-basead cross-sectional survey. International Journal of Environmental Research and Public Health, 17(5933), 1-14. https://doi.org/10.3390/ijerph17165933

Budak, M., Kahramanoglu, I., Vitale, S., Akgol, S., Dilek, M., Kartal, S. et al. (2019). Maternal abdominal subcutaneous fat thickness as a simple predictor for gestational diabetes mellitus. Journal of Perinatal Medicine, 47(6):605-610.

Ceulemans, M., Hompes, T. \& Foulon, V. (2020, julho). Mental health status of pregnant and breastfeeding women during the COVID-19 pandemic: a call for action. International Journal of Gynecology \& Obstetrics, 151, 146-147. https://doi.org/10.1002/ijgo.13295

Christian, L., Carroll, J. E., Teti, D. \& Hall, M. (2019). Maternal sleep in pregnancy and postpartum part I: mental, physical, and interpersonal consequences. Current Psychiatry Reports, 21(20), 1-8. https://doi.org/10.1007/s11920-019-0999-y

Dennis, C. L., Falah-Hassani, K. \& Shiri, R. (2017, maio). Prevalence of antenatal and postnatal anxiety: systematic review and meta-analysis. British Journal of Psychiatry, 210(5), 315-323. 10.1192/bjp.bp.116.187179.

Dong, H., Hu, R., Lu, C., Huang, D., Cui, D., Huang, G. et al. (2020, outubro). Investigation on the mental health status of pregnant women in China during the pandemic of COVID-19. Archives of Gynecology and Obstettrics, 303, 463-469. 10.1007 / s00404-020-05805-x 
Estrela, F. M., Silva, K. K., Cruz, M. A. \& Gomes, N. P. (2020). Gestantes no contexto da pandemia da Covid-19: reflexões e desafios. Physis: Revista de Saúde Coletiva, 30(2):1-5

Fan, S., Guan, J., Cao, L., Wang, M., Zhao, H., Chen, L. et al. (2021, fevereiro). Psychological effects caused by COVID-19 pandemic on pregnant women: a systematic review with meta-analysis. Asian Journal of Psychiatry, 56, 102533. https://doi.org/10.1016/j.ajp.2020.102533

Figueiroa, M., Menezes, M., Monteiro, E., Aquino, J., Mendes, N. \& Silva, P. (2017). Acolhimento do usuário e classificação de risco em emergência obstétrica: avaliação da operacionalização em maternidade-escola. Escola Anna Nery, 21(4), e20170087. https://doi.org/10.1590/2177-9465-EAN-2017-0087

Führer, A., Frese, T., Karch, A., Mau, W., Meyer, G., Richter, M. et al. (2020, agosto). COVID-19: knowledge, risk perception and strategies for handling the pandemic. Journal of Evidence in Health Care, 153-154, 32-38. https://doi.org/10.1016/j.zefq.2020.06.002

Gonçalves, L. A., Monteiro, C. F., Silva Júnior, F., Veloso, L. U., Oliveira, A. D. \& Nunes, B. M. (2020). Rastreio do consumo de bebidas alcoólicas em gestantes. Reme: Revista Mineira de Enfermagem, 24(e1322), 1-6. https://dx.doi.org/10.5935/1415-2762.20200059

Grigoriadis S., Graves, L., Peer, M., Mamisashvili, L., Tomlinson, G., Vigod, S., et al. (2018, setembro). Maternal anxiety during pregnancy and the association with adverse perinatal outcomes: systematic review and meta-analysis. Journal of Clinical Psychiatry, 79(5), 17r12011, 10.4088/JCP.17r12011

Lebel, C., MacKinnon, A., Bagshawe, M., Tomfohr-Madsen, L. \& Giesbrecht, G. (2020, abril). Elevated depression and anxiety among pregnant individuals during the COVID-19 pandemic. PsyArxiv Preprints, 277, 5-13. 10.31234/osf.io/gdhkt

Lee R. W., Loy, S. L., Yang, L., Chan, J. K. \& Tan, L. K. (2020). Attitudes and precaution practices towards COVID-19 among pregnant women in Singapore: a cross-sectional survey. BMC Pregnancy and Childbirth, 20(675), 1-10. https://doi.org/10.1186/s12884-020-03378-w

Lucci, T. K., Otta, E., David, V. F., \& Chelini, M. O. M. (2016). Depressão materna e concentração de cortisol de recém-nascidos em uma amostra brasileira. Revista Psico, 47(2), 140-147. http://dx.doi.org/10.15448/1980-8623.2016.2.23655

Matvienko-Sikar, K., Pope, J., Cremin, A., Carr, H., Leitao, S., Olander, E. K. et al. (2020, outubro). Differences in levels of stress, social support, health behaviours, and stress-reduction strategies for women pregnant before and during the covid-19 pandemic, and based on phases of pandemic restrictions, in Ireland. Women and Birth, 1207, 1-8. 10.1016/ j.wombi.2020.10.010

Pancani, L., Marinucci, M., Aureli, N. \& Riva, P. (2020, setembro). Forced social isolation and mental health: a study on 1006 italians under COVID-19 lockdown. Arquivo de Psicologia, 1-22. 10.31234/osf.io/uacfj

Parra-Saavedra, M., Villa-Villa, I., Pérez-Olivo, J., Guzman-Polania, L., Galvis-Centurion, P., Cumplido-Romero, Á. et al. (2020, novembro). Attitudes and collateral psychological effects of COVID-19 in pregnant women in Colombia. International Journal of Gynecology \& Obstetrics, 151(2):203-208. https://doi.org/10.1002/ijgo.13348

Pereira A. S. et al. (2018). Metodologia da pesquisa científica. UFSM.

Rodríguez-Bernal, C. L., Ramón, R., Quiles, J., Murcia, M., Navarrete-Muñoz, E., Vioque, J. et al. (2013). Dietary intake in pregnant women in a Spanish mediterranean área: as good as it is supposed to be? Public Health Nutrition, 16(8), 1379-1389.

Salman, H., Shah, M., Ali, A., Aziz, A., \& Vitale, S. (2018, julho). Assessment of relationship of sérum neurokinin- B level in the pathophysiology of preeclampsia: a case control study. Advances in Therapy, 35(7), 1114-1121.

Silva, M., Nogueira, D., Clapis, M. \& Leite, E. (2017). Ansiedade na gravidez: prevalência e fatores associados. Revista da Escola de Enfermagem da USP, 51, e03253. https://doi.org/10.1590/S1980-220X2016048003253

Stepowicz, A., Wencka, B., Bieńkiewicz, J., Horzelski, W. \& Grzesiak, M. (2020, dezembro). Stress and anxiety levels in pregnant and post-partum women during the COVID-19 pandemic. Internacional Jornal of Environmental Research and Public Health, 17(24), 9450. https://doi.org/10.3390/ijerph17249450

Thapa, S. B., Mainali, A., Schwank, S. E. \& Acharya, G. (2020, maio). Maternal mental health in the time of the COVID-19 pandemic. Acta Obstetricia et Gynecologica Scandinavica, 99(7), 817-818. https://doi.org/10.1111/aogs.13894

Wang, C., Pan, R., Wan, X., Tan, Y., Xu, L., Ho, C. et al. (2020, março). Immediate psychological responses and associated factors during the initial stage of the 2019 coronavirus disease (COVID-19) epidemic among the general population in China. International Journal of environmental Research and Public Health, 17(5), 1729 .

Yan, H., Ding, Y., \& Guo, W. (2020, novembro). Mental health of pregnant and postpartum women during the coronavirus disease 2019 pandemic: a systemactic review and meta- analysis. Frontiers in Psychology, 11(617001), 1-12. https://doi.org/10.3389/fpsyg.2020.617001

Yılmaz, C. \& Gökmen, V. N. (2020, fevereiro). Neuroactive compounds in foods: ocorrence, mechanism and potential health effects. Food Research International, 128, 108744. 